\title{
Assisting Database Users in a Web Environment
}

\author{
Silvia Schiaffino ${ }^{1,2}$ and Analía Amandi ${ }^{1}$ \\ ${ }^{1}$ ISISTAN Research Institute - Univ. Nac. del Centro Pcia. Bs. As. \\ Campus Universitario, Tandil, 7000, Bs. As., Argentina \\ 2 Also CONICET \\ \{sschia, amandi@exa.uncen.edu.ar\}
}

\begin{abstract}
Intelligent agents assisting users with repetitive tasks learn how these users perform these tasks and then they help users by making suggestions and, sometimes, by executing tasks on behalf of the users. Making queries to a relational database system is a repetitive and time-consuming task in which intelligent agents can be very helpful. In this work we present QueryGuesser, an intelligent agent with the capability of generating personalized queries according to a user's working habits and information needs. This agent observes a user's behavior while he is working with a database system and builds the user's profile. This profile is used to generate and execute personalized queries in advance, reducing the amount of time the user has to wait for the answers and offering the user the information he needs before he has to ask the system for it.
\end{abstract}

\section{Introduction}

The revolution in technologies and communications we have been experiencing in the last decade has left us a great volume and variety of information available in different electronic media. A big part of this information is recorded in relational databases, which are manipulated by information systems through query languages. With the growing of the WWW several companies have leveraged their database systems to Internet and Intranet contexts. Managing the information contained in databases in these new contexts has become a progressively more complex process that demands many time and effort from users [1].

In this context consider, for example, those users who regularly make queries to a given database system (within an Intranet or via Web) searching for information relevant to their interests. For example, consider a set of technicians working at a laboratory in a petrochemical plant. These employees are in charge of tracking and analyzing information about samples of different products. This information is stored in the laboratory database system. In their daily work, these technicians make queries to this database to get the information they need. Making queries is a time-consuming activity for these users, since they generally have difficulties in getting the information they need in a rapid manner due to network connections and server delays and they have to wait for it more than they would want to. Making queries is also a routine task since these users make similar queries in different opportunities to get the information they 
usually work with. In consequence, users spend their time performing repetitive and time-consuming tasks instead of working productively with these information systems.

In this work we present an intelligent agent that acts as a personal assistant of a database user. This agent, named QueryGuesser, is capable of determining the queries a user commonly executes when he has certain information needs. Then, our agent can assist the user with his database work by suggesting him the execution of queries and by executing some of these queries on the user's behalf.

In order to achieve its goal, our agent has to solve some problems. First, it has to learn which queries the user generally makes to obtain information relevant to his interests and information needs. Second, the agent has to learn when the user executes each type of query, i.e. if there exist some kind of behavioral pattern in the user's actions. Finally, the agent has to detect changes in both a user's interests and working habits.

Our agent attacks these problems by utilizing a technique we have developed to build user profiles that combines Case-Based Reasoning (CBR) and Bayesian Networks (BN). QueryGuesser observes a user's behavior while he is making queries and records each querying experience as a case. Those cases representing similar queries are clustered in order to determine the various information needs the user has and his different querying habits. The agent uses the information contained in the case base to build a BN that models the relationships among query features. The BN allows the agent to deal with uncertain information about the user's information needs and the relationships among them.

This paper is organized as follows. Section 2 presents an overview of QueryGuesser. Section 3 describes some experimental results. Finally, Section 4 presents our conclusions.

\section{The QueryGuesser Agent: An Overview}

QueryGuesser is an intelligent agent that assists users who work with a relational database system through Web or Intranet applications. The QueryGuesser agent has the capability of managing personalized queries according to the information needs (or interests) and working habits of a given user. QueryGuesser's goal is to obtain this information about a user from the queries he generally makes. Once the agent has obtained this information, it can assist the user with the operation of the database.

QueryGuesser can help the user in two ways: it can suggest the user the execution of queries according to his interests and needs, and it can perform some of these queries in advance in order to save the user's time. Then, the agent processes the feedback the user provides regarding the assistance it has given him. The user can explicitly evaluate the quality and relevance of the queries the agent has suggested him and the behavioral patterns the agent has inferred. Besides, the agent can observe the user's behavior after assisting him to detect some indirect (or implicit) feedback, such as the user making a query different to the one suggested by the agent.

In order fulfill its tasks, a QueryGuesser agent must have the capability of building a user profile, which represents the user's habits and information needs in a given 
database domain. These information needs or interests are represented by the attributes or keywords involved in the user's queries. Thus, each topic of interest is described by a set of attributes and the values these attributes take, as well as an importance value or weight associated with each attribute and attribute value. The user's working habits are described by a set of situations in which the user makes queries. Each situation is associated with one or more topics of interest, and vice versa. For example, a situation may indicate that a user makes a certain type of query every morning or at the end of the month.

QueryGuesser learns about a user by observing his behavior while he is working with a database application and by recording data obtained from this observation process. This information is stored in the form of cases, according to the CBR paradigm. CBR means reasoning based on previous cases or experiences [3]. In CBR, a reasoner remembers previous situations similar to the current one and uses them to solve a new problem. In this work, each case records information about a particular user query, i.e. the attributes or keywords used by the user to make the query and temporal information describing the moment in which the query was executed (e.g. date, time, shift). We use interpretive CBR to classify a new query according to its similarity with previous recorded ones. Similar queries are clustered, forming different topics of interest. The agent classifies the queries considering two different criteria: content similarity and temporal information similarity.

In addition to storing a user's queries in a case base, the agent also builds a Bayesian model of the user's interests. A BN models the relationships among items of interest and the strength of these relationships. A BN is a directed acyclic graph that represents a probability distribution, where nodes represent random variables and arcs represent probabilistic correlation between variables [2]. The $\mathrm{BN}$ is dynamically updated as new information is obtained from the observation of the user's behavior. The information stored in the case base and in the $\mathrm{BN}$ of a given user is used to build the user profile.

\section{Experimental Results}

We implemented the QueryGuesser agent in Java and we used JavaBayes ${ }^{1}$ to manipulate the BN. The QueryGuesser agent was tested while assisting a set of 20 users that operate the subsystem in charge of sample tracking within a LIMS (Laboratory Information Management System). The main goal of these tests was to analyze if the content of user profiles improved as the agent interacts with the user, tending to the user's needs. We studied the effects of user feedback in the profile building process, since we can view the evolution of user profiles in this way.

In order to make the tests, we asked each user to make twenty queries. At certain points, which were different for each user, the user required the agent's assistance.

\footnotetext{
${ }^{1}$ http://www.cs.cmu.edu/ javabayes/Home
} 


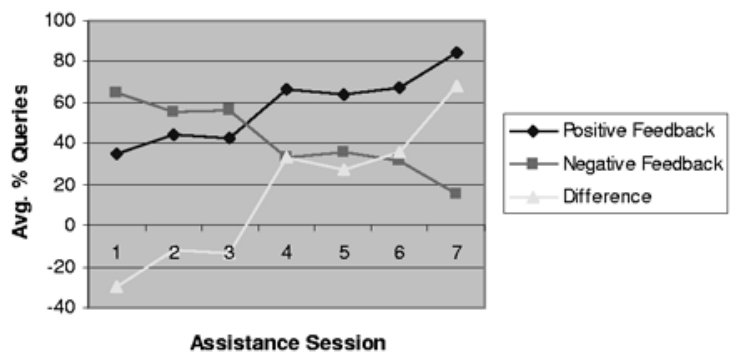

Fig. 1. Evolution of User Profiles

Then, we asked each user to provide feedback for each query suggested by the agent, rating them as relevant or irrelevant. The graph in Figure 1 plots the performance of the QueryGuesser agent. The graph plots, for each session, the average percentage of suggested queries that received positive feedback, the average percentage of suggested queries that received negative feedback and the difference between them. We can observe that there is a pattern of improving performance in the queries suggested by the agent. We can observe a soft learning curve in the initial states, since the first feedback values are mostly negative. This situation arose because we did not wait until the agent had learned something about the users' preferences to start testing it. In this way, the agent gave assistance to the user having information about a few queries made by the users. But then, due to the users' feedback, the agent stopped suggesting general (irrelevant) queries and feedback values became positive.

\section{Conclusions}

In this paper, we have presented QueryGuesser, an agent developed to assist users in their work with a database system. Our agent has been successfully tested in a LIMS, assisting users who made queries from distant places in a petrochemical plant. These users had considerable delays in getting the information they needed. Thus, our agent was very useful to them. The experimental results obtained so far have proved that our agent is good (and promising) at determining a user's topics of interest.

\section{References}

1. Y. Han and L. Sterling: Agents for citation finding on the World Wide Web. In PAAM 97, pages 303-318, 1997

2. J. Kolodner. Case-Based Reasoning. Morgan Kaufmann Publishers, 1993

3. P. Haddawy. An overview of some recent developments in Bayesian problem-solving techniques. AI Magazine, pages 11-19, 1999 